CORRIGENDUM

doi:10.1038/nature21730

\title{
Corrigendum: Carcinoma-astrocyte gap junctions promote brain metastasis by cGAMP transfer
}

Qing Chen, Adrienne Boire, Xin Jin, Manuel Valiente, Ekrem Emrah Er, Alejandro Lopez-Soto, Leni S. Jacob, Ruzeen Patwa, Hardik Shah, Ke Xu, Justin R. Cross \&

Joan Massagué

Nature 533, 493-498 (2016); doi:10.1038/nature18268

In this Article, Extended Data Figs 7d and 9a, presenting results obtained with the H2030-BrM3 cell line, contain errors that were introduced during the assembly of these figures in parallel with the corresponding panels in Figs $3 \mathrm{~b}$ and $4 \mathrm{a}$, which show the results of the same experiment but using a different cell line (MDA231-BrM2). The p65 loading control from Fig. $3 \mathrm{~b}$ and the TBK1 loading control from Fig. 4a were duplicated in Extended Data Figs 7d and 9a, respectively. The STAT1 strip in Extended Data Fig. 7d was horizontally reversed. The Supplementary Information to this Corrigendum contains the revised Extended Data Fig. 7d (with the correct STAT1 and p65 strips) and the revised Extended Data Fig. 9a (with the correct TBK1 strip) for the H2030-BrM3 cell line as well as full scans of all western blots for Extended Data Figs 1-10, which were not originally provided. These errors do not alter the results or conclusions of the Article. The original Article has not been corrected online.

Supplementary Information is available in the online version of the Corrigendum. 